\title{
A Study on the Attention of Yoga and Its Development Based on Complex Network Theory
}

\author{
Pengchao Li $^{1}$, Qinghong Miao ${ }^{2 *}$, Yuchi Meng ${ }^{3}$, Jie Ning ${ }^{4}$, Jing Long ${ }^{5}$ and Junya Huang ${ }^{1 *}$ \\ ${ }^{1}$ School of Art, Beijing Sport University, Beijing, China, ${ }^{2}$ Beijing Jingkong Technology Co., Ltd., Beijing, China, ${ }^{3}$ Chinese Skating \\ Association, Beijing, China, ${ }^{4}$ School of Physical Education, Zhoukou Normal University, Zhoukou, China, ${ }^{5}$ Zhongshan \\ Experimental Middle School, Zhongshan, China
}

Taking Baidu search index as the data source, this research collects yoga-related data in various provinces in China, analyzes the public's attention to yoga on the Internet from the perspective of the complex network theory, so as to dig out characteristics of those who are interested in yoga as well as the temporal and spatial change of yoga attention from 2011 to 2020. Then, by transforming the time series into a network through the VG model and HVG model, the paper analyzes the network characteristics and predicts the popularity of yoga. Furthermore, the publicity of yoga and the public's attention to it

OPEN ACCESS

Edited by:

Peican Zhu,

Northwestern Polytechnical University, China

Reviewed by:

Mingyang Zhou,

Shenzhen University, China

Wei Wang,

Sichuan University, China

${ }^{*}$ Correspondence:

Qinghong Miao

miaoqh_1101@163.com

Junya Huang

junyahuang@sina.com

Specialty section:

This article was submitted to

Social Physics,

a section of the journal

Frontiers in Physics

Received: 01 June 2021

Accepted: 26 July 2021

Published: 01 October 2021

Citation:

Li P, Miao Q, Meng Y, Ning J, Long J and Huang $J$ (2021) A Study on the Attention of Yoga and lts Development Based on Complex Network Theory.

Front. Phys. 9:719012.

doi: 10.3389/fphy.2021.719012 are analyzed, considering the policy, national education level, the influence of $\mathrm{TV}$, mobile phone and other communication equipment, so as to help the market to provide corresponding products and services in a targeted manner and to promote the healthy development of the yoga industry.

Keywords: complex network, social network, yoga, attention, visualization graph

\section{INTRODUCTION}

In recent years, the government has been vigorously developing national fitness. The "Healthy China 2030" Planning Outline and the "Opinions on Promoting National Fitness and Sports Consumption to Promote the High-quality Development of the Sports Industry" issued by the Party Central Committee and the State Council both recognize the necessity of carrying out national fitness campaign, activating the fitness training market and building a better platform for the sports industry. Yoga is a physical, mental, and spiritual exercise with a history of more than 5,000 years and it represents "harmony" and "consistency". Its essence is to connect "self" and "superego," to transform the soul and to improve physical and mental health. Since the 19th century, modern yoga has been attracting groups of all ages rapidly with its functions, such as improving posture and self-cultivation. It is estimated that there were 2.5 million yoga practitioners in the United Kingdom and 15 million in the US in 2008 [1], and the number has been increasing rapidly in recent years.

At present, the number of researches on yoga at home and abroad has been increasing significantly year by year. Though its research content covers the history of yoga, the feasibility of introducing yoga and the efficacy of yoga, the majority of them are purely theoretical description and analysis based on transplantation of related theories and adoption of conceptual introduction and explanatory methods. These researches focus on theoretical explanations and abstract categories, featuring low-level repeated studies and weakness of absorbing new research methods. The relationship between yoga and human health is one of the important directions of domestic research. Wang Min (2005) conducted a 15 weeks experiment on female college students, showing that yoga practice can effectively improve their respiratory system, circulatory system 
skills, physical flexibility, balance ability, and mental health [2]. Wu Minyue (2010) reviewed scientific research papers on yoga and health at home and abroad, showing that long-term insistent practice of yoga has a positive influence on preventing and treating chronic diseases such as cardiovascular diseases and diabetes [3]. Over time, the medical field has recognized the efficacy of yoga and has been adopting it into the treatment and management of diseases, including chronic disease [4], depression [5-7], cancer [8, 9], and rehabilitation. Later, the study of yoga is no longer restricted to its exercising and health function and covers the development of the entire industry, causing an in-depth impact on yoga research. Huang Min (2010), Chen Xiaoying (2010), and some other researchers analyzed the development status of yoga-related industries from the perspective of yoga industrialization and marketization and put forward suggestions for the sustainable development of yoga industry [10, 11]. Yu Jingjing (2011), Liu Min (2013), and Zhang Maomao (2014) discussed the social background and value orientation of yoga and the building of yoga teaching teams in universities [12-14]. VG model is also used in stock and venture capital $[15,16]$.

Compared with domestic research, foreign research presents the characteristics of overlap, penetration, and integration of multidiscipline. It pays special attention to the adoption of empirical research methods such as randomized controlled trials, double-blind trial intervention, meta-analysis, follow-up, prospective studies, and nationwide surveys. The research content is systematic and target-orientated. For instance, Cramer H (2013) analyzed 12 randomized controlled trials (619 participants) to study the relationship between yoga and its influence on relieving depression and anxiety and improving the quality of life. He argues that yoga can be considered as one of the adjunctive treatment methods for patients with depression or individuals with elevated levels of depression [17]. Stemlieb B (2011) conducted a randomized controlled experimental intervention on breast cancer patients, discovering that targeted yoga intervention can significantly improve the persistent fatigue symptoms [18]. Bussing A (2012) believes that although yoga has not been proven to be an independent treating method due to some research conditions, the beneficial effects of yoga intervention on physical and mental health related to pain do exist. As adjunctive therapy, yoga can improve body function and self-confidence [19]. With the widespread concept of practicing yoga, more research has been conducted on the relations between yoga and the treatment of diseases including obesity [20,21], chronic blood disease [22], mental health $[23,24]$, cancer $[25,26]$, and even COVID 19 prevention $[27,28]$. The increasing popularity of yoga can be partly attributed to the Internet and social media. Social media bloggers have been attracting more and more yoga practitioners. Chen et al. (2014) designed a yoga pose recognition model to help yoga practitioners to practice with more appropriate postures. The Internet also provides researchers a better platform to conduct their studies. K Firestone (2014), Johnson (2014) conducted online surveys to collect data [29, 30], which has some similarities to our study.

This paper breaks the boundaries of domestic literature, adopts search behavior data to measure public's attention on yoga, and attempts to investigate the time change trend of yoga attention in a specific time and space environment and the characteristics of the practitioner, so as to understand the public's need for yoga and its changing trend and analyze the evolution of yoga attention by establishing the time series prediction model of yoga attention degree, so as to propose suggestions on the future yoga development directions and strategies. The second part of the paper is a description of the methods used. The third part is a complex network-based analysis and visualization of the Baidu search index data of yoga from 2011 to 2020 . The fourth part is the prediction of the popularity of yoga and relevant suggestions.

\section{MODEL DESCRIPTION}

\section{Data Extraction and Processing}

Attention, which refers to the degree to which a thing receives interests from social groups, is an important indicator of the strength of the internal relationship between things and group behavior, exerting an important influence on public opinion, culture, and policy. Attention can be reflected by different parameters, such as the number of searches, views, reposts, comments, and favorites of an event on the Internet. Based on Baidu's massive data, the Baidu index, on the one hand, analyzes the hotness of keywords and, on the other hand, explores in-depth the data characteristics of public opinion, market demand, and user characteristics. The Baidu index reflects the active search demands of internet users, and all activities that affect their search behavior may affect the Baidu index. Therefore, in order to show the public's attention to yoga and those who are interested in yoga, this study selects the visualization results of the Baidu index search as the data basis for analyzing domestic users' attention to yoga. The interpolation method is adopted to obtain equal interval data in case missing sampling or uneven sampling interval of the data occurs.

\section{Modeling of Complex Networks}

A complex network describes a system composed of a large number of interacting individuals. The analysis of a complex network can help describe the structure of the network system and understand the law of information evolution on the network, so as to finally realize the intervention and optimization of information evolution process on the network. This paper uses the time series visualization method proposed by Lacasa et al. [31] to construct the network, which consists of the Baidu search index time series subsystem with the keyword "yoga" in 31 provinces in China. First, the discrete time series data of the subsystem $X(t)$ corresponds to the nodes of the network, and the connection edges are constructed according to the visual criterion. The connection edge can be established by 

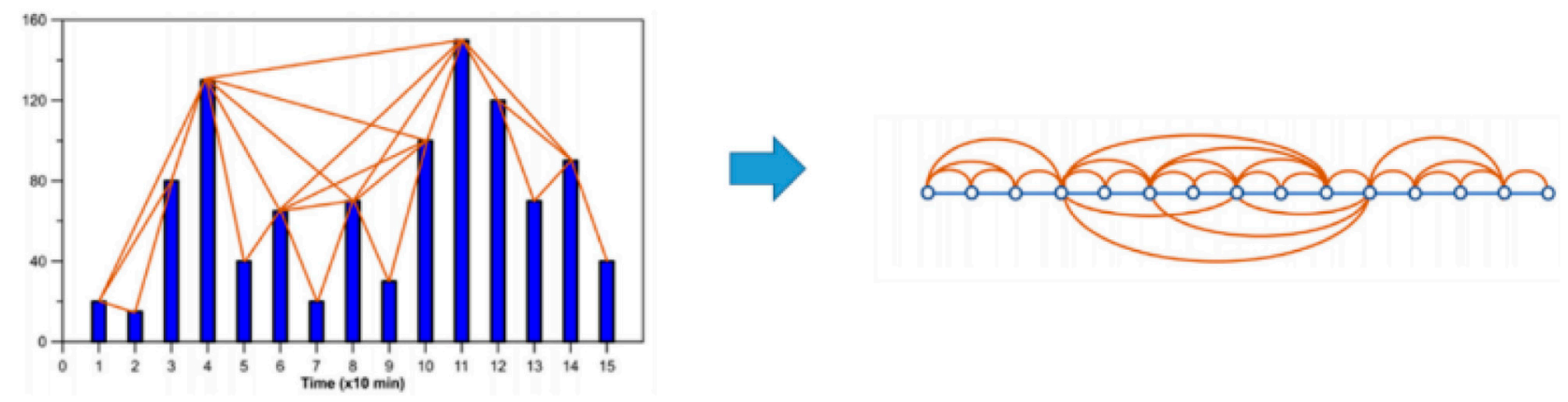

FIGURE 1 | The theory of time series data visualization.

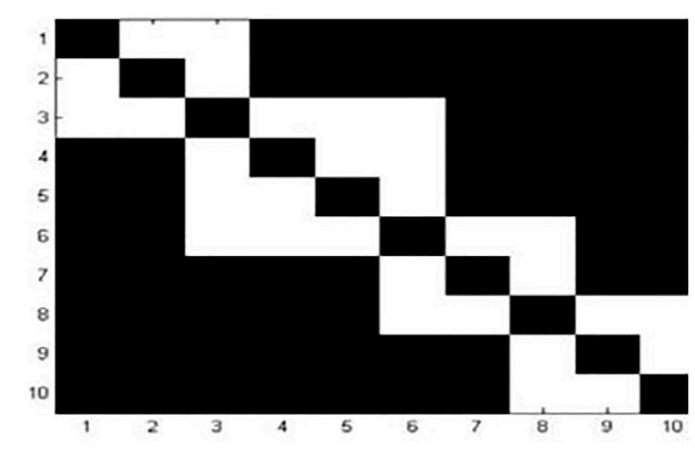

邻挨短阵

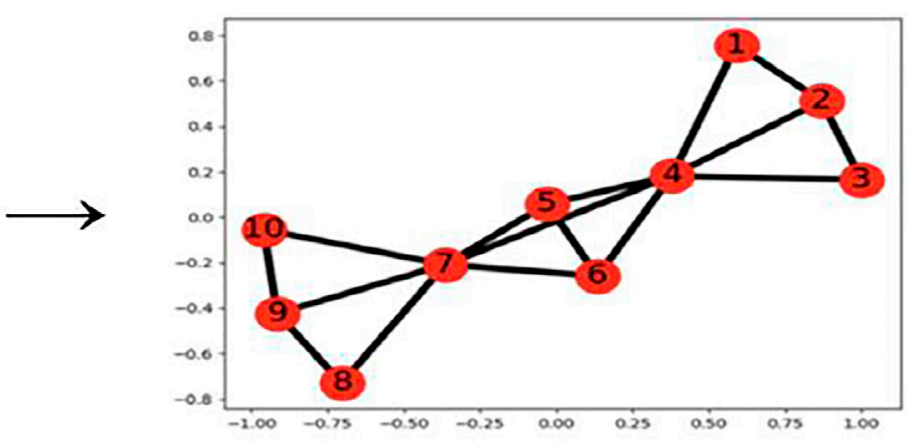

网络图

FIGURE 2 | Visual network construction process and characteristics extraction.

visualizing the data of any two points. In the time series $(X(t))$ any point between $\left(t^{a}, \mathrm{x}^{a}\right)$ and $\left(t^{c}, \mathrm{x}^{c}\right)$ can be linked, and when $t^{a}<t^{b}<t^{c}$ any point $t^{b}, t^{b}$ between $t^{a}, t^{a}$ and $t^{c}, t^{c}$, all satisfy:

As shown in Figure 1, the height of the bar represents the data value at each time point. If the tops of the 2 bars are visible to each other, the corresponding two points are connected by the network in the figure.

Secondly, to construct an adjacency matrix based on time series nodes and edges, and to form a network graph, as shown in Figure 2.

The important characteristics of the complex network are calculated as follows [32-34]:

(1) Degree and degree distribution.

The number of edges connected by a node is called the degree of the node, the node $\mathrm{i}$ is shown as $\mathrm{k}_{\mathrm{i}}$ :

$$
K_{i}=\sum_{j} a_{i j}
$$

Among them, $a_{i j}$ is the number of connecting edges between nodes $i$ and $j$. In the network, the greater the degree, it means the more nodes are connected to it, then the greater influence of the node, and thus the stronger time correlation. The average degree is defined as follows:

$$
K=\frac{1}{N} \sum_{i} k_{i}
$$

Among them, $\mathrm{N}$ represents the number of nodes in the network.

(2) Average path length and diameter.

The path connecting two reachable nodes in the network with the least number of edges is called the distance between nodes, and the longest path between two nodes is called the diameter of the network $\mathrm{d}_{\mathrm{ij} \text {. }}$ Both the average network path length and the network diameter can reflect the network transmission efficiency. The smaller the value, it means that the transmission effect can be achieved with fewer nodes in the network, thus the higher network efficiency. The calculation formula for the average network path length is as follows:

$$
L=\frac{1}{\frac{1}{2} N(N-1)} \sum_{i \geq j} d_{i j}
$$

(3) Clustering coefficient and clustering.

The clustering coefficient, which describes the degree of clustering of all nodes in the network, represents the tightness of the network. Its calculation formula is as follows:

$$
C_{i}=\frac{2 E_{i}}{K_{i}\left(K_{i}-1\right)}
$$


TABLE 1 | Descriptive statistics of Baidu Index of Yoga in 2020.

\begin{tabular}{|c|c|c|c|c|c|c|c|c|c|c|c|c|}
\hline \multirow[b]{2}{*}{ Province } & \multicolumn{3}{|c|}{ Average } & \multicolumn{3}{|c|}{ Maximum } & \multicolumn{3}{|c|}{ Minimum } & \multicolumn{3}{|c|}{ Standard error } \\
\hline & $\begin{array}{c}\text { Search } \\
\text { index }\end{array}$ & PC & Mobile & $\begin{array}{l}\text { Search } \\
\text { index }\end{array}$ & PC & Mobile & $\begin{array}{l}\text { Search } \\
\text { index }\end{array}$ & PC & Mobile & $\begin{array}{l}\text { Search } \\
\text { index }\end{array}$ & PC & Mobile \\
\hline Anhui & 290.9559 & 49.1176 & 241.8382 & 441 & 74 & 367 & 136 & 0 & 136 & 85.3160 & 24.3436 & 70.2625 \\
\hline Macau & 7.6176 & 0.0000 & 7.6176 & 60 & 0 & 60 & 0 & 0 & 0 & 19.6522 & 0.0000 & 19.6522 \\
\hline Beijing & 437.1618 & 69.8676 & 367.2941 & 592 & 101 & 518 & 181 & 0 & 181 & 106.9644 & 18.3107 & 96.2235 \\
\hline Fujian & 260.2794 & 52.3088 & 207.9706 & 350 & 78 & 287 & 144 & 0 & 123 & 57.8735 & 22.3395 & 46.2014 \\
\hline Gansu & 175.6471 & 31.6029 & 144.0441 & 250 & 63 & 205 & 89 & 0 & 89 & 53.1994 & 29.1722 & 33.0266 \\
\hline Guangdong & 704.0588 & 79.9412 & 624.1176 & 992 & 142 & 858 & 348 & 57 & 287 & 184.4647 & 16.2957 & 174.1870 \\
\hline Guangxi & 238.5000 & 50.3971 & 188.1029 & 339 & 70 & 278 & 115 & 0 & 115 & 51.5927 & 22.4839 & 40.7710 \\
\hline Guizhou & 163.8676 & 22.2941 & 141.5735 & 269 & 63 & 208 & 92 & 0 & 83 & 47.0779 & 28.5689 & 30.2188 \\
\hline Hainan & 128.1618 & 26.5147 & 101.6471 & 191 & 63 & 139 & 67 & 0 & 67 & 37.8889 & 29.2026 & 18.7464 \\
\hline Hebei & 501.1912 & 59.4706 & 441.7206 & 710 & 85 & 683 & 208 & 0 & 206 & 167.9120 & 19.9046 & 158.7823 \\
\hline Henan & 491.6029 & 58.9559 & 432.6471 & 726 & 89 & 656 & 201 & 0 & 201 & 166.1601 & 19.4778 & 158.3369 \\
\hline Heilongjiang & 274.8971 & 49.2647 & 225.6324 & 403 & 70 & 337 & 138 & 0 & 119 & 77.7641 & 24.3743 & 66.9417 \\
\hline Hubei & 335.6912 & 56.5000 & 279.1912 & 527 & 76 & 453 & 128 & 0 & 128 & 104.3891 & 21.1861 & 93.0765 \\
\hline Hunan & 281.3529 & 46.5000 & 234.8529 & 398 & 80 & 324 & 148 & 0 & 148 & 74.3916 & 27.3777 & 59.5925 \\
\hline Jilin & 225.8235 & 43.5147 & 182.3088 & 333 & 74 & 267 & 92 & 0 & 92 & 62.9944 & 27.4783 & 44.9006 \\
\hline Jiangsu & 468.7059 & 65.9118 & 402.7941 & 667 & 104 & 591 & 232 & 0 & 201 & 125.1420 & 21.4293 & 111.7364 \\
\hline Jiangxi & 247.9412 & 47.8235 & 200.1176 & 387 & 78 & 326 & 109 & 0 & 109 & 68.6181 & 24.7844 & 54.2321 \\
\hline Liaoning & 345.2206 & 59.3088 & 285.9118 & 479 & 80 & 419 & 157 & 0 & 157 & 87.5737 & 15.8060 & 80.3566 \\
\hline $\begin{array}{l}\text { Inner } \\
\text { mongolia }\end{array}$ & 216.2794 & 41.1618 & 175.1176 & 337 & 66 & 278 & 95 & 0 & 95 & 67.1258 & 27.8233 & 49.5706 \\
\hline Ningxia & 97.8235 & 11.0147 & 86.8088 & 176 & 61 & 121 & 63 & 0 & 63 & 30.6876 & 22.8307 & 15.5604 \\
\hline Qinghai & 85.3529 & 9.4559 & 75.8971 & 153 & 61 & 100 & 57 & 0 & 57 & 26.4551 & 21.6963 & 10.4869 \\
\hline Shandong & 636.7206 & 69.0000 & 567.7206 & 910 & 110 & 825 & 312 & 0 & 255 & 196.8528 & 15.6205 & 188.8028 \\
\hline Shanxi & 286.9412 & 50.5000 & 236.4412 & 395 & 83 & 338 & 125 & 0 & 125 & 82.4659 & 23.9574 & 69.7311 \\
\hline Shaanxi & 295.4706 & 58.4559 & 237.0147 & 401 & 70 & 337 & 125 & 0 & 119 & 72.0341 & 13.1324 & 65.0191 \\
\hline Shanghai & 288.7794 & 63.2794 & 225.5000 & 374 & 104 & 313 & 122 & 0 & 122 & 51.5004 & 15.9382 & 42.4276 \\
\hline Sichuan & 410.0882 & 59.6029 & 350.4853 & 587 & 80 & 521 & 186 & 0 & 186 & 120.6645 & 17.8494 & 110.3189 \\
\hline Taiwan & 16.0147 & 3.3529 & 12.6618 & 115 & 57 & 58 & 0 & 0 & 0 & 29.4454 & 13.5115 & 23.9786 \\
\hline Tianjin & 197.6324 & 40.4118 & 157.2206 & 282 & 70 & 219 & 86 & 0 & 86 & 52.4248 & 28.2691 & 33.5936 \\
\hline Tibet & 63.1324 & 2.5735 & 60.5588 & 126 & 61 & 78 & 0 & 0 & 0 & 17.3609 & 12.0748 & 15.9658 \\
\hline Hongkong & 53.5441 & 5.0882 & 48.4559 & 120 & 59 & 69 & 0 & 0 & 0 & 30.9542 & 16.4804 & 23.8924 \\
\hline Xinjiang & 189.0441 & 36.1912 & 152.8529 & 269 & 64 & 212 & 99 & 0 & 99 & 51.2443 & 28.7341 & 35.3428 \\
\hline Yunnan & 199.7059 & 31.1765 & 168.5294 & 291 & 66 & 227 & 109 & 0 & 109 & 53.6976 & 29.6668 & 34.0910 \\
\hline Zhejiang & 403.8824 & 64.1176 & 339.7647 & 537 & 87 & 469 & 200 & 0 & 179 & 97.2186 & 19.8709 & 86.1114 \\
\hline Chongqing & 223.0882 & 44.2353 & 178.8529 & 307 & 78 & 249 & 102 & 0 & 102 & 60.4621 & 26.9400 & 42.2273 \\
\hline
\end{tabular}

Among them, $C_{i}$ represents the clustering coefficient of node $i, K_{i}$ represents the number of nodes connected to node $i$, and $E_{i}$ represents the actual number of edges between nodes in the network. It can be deduced that the average clustering coefficient $\mathrm{C}$ of the network is as follows:

$$
C=\frac{1}{N} \sum_{i=1}^{N} C_{i}
$$

The value of the clustering coefficient is generally between 0 and 1 . The larger the coefficient, the better the connectivity of the network and the greater the degree of network aggregation. In general, some real-world network clustering coefficients are larger than random network clustering coefficients of the same size, which means that real networks have better clustering tendency, which features the nature of clustering.

(4) Density.

The density of the network is equal to the ratio of the actual number of edges in the network to the maximum possible number of edges in the network. The greater the density, the more the number of edges in the network and the denser the network.

\section{EMPIRICAL ANALYSIS}

We analyze the time series data of the Baidu index for 31 provinces and cities from 2011 to 2020. The descriptive statistics, visualization of time series data, and complex network method are also applied to the analysis to find out the characteristics of the visualized networks.

\section{Descriptive Statistics}

As showed in Table 1, based on the search result of "yoga" in the Baidu index, Guangdong, Shandong, Hebei, Henan, and Jiangsu are the top 5 provinces that pay the most attention to yoga. These five are all provinces with large population and economy. But we noticed that the standard error of the index in these provinces is in the top position too. It is found that the data of these provinces are more prone to fluctuations due to Internet searches. Figure 3 shows the variation tendency of the Baidu index of the top 5 and the bottom 3 provinces in 2020. The increase of the Baidu index in 2021 is, on the one hand, due to the COVID-19 pandemic, which brings exercises and health issues to the public's attention 

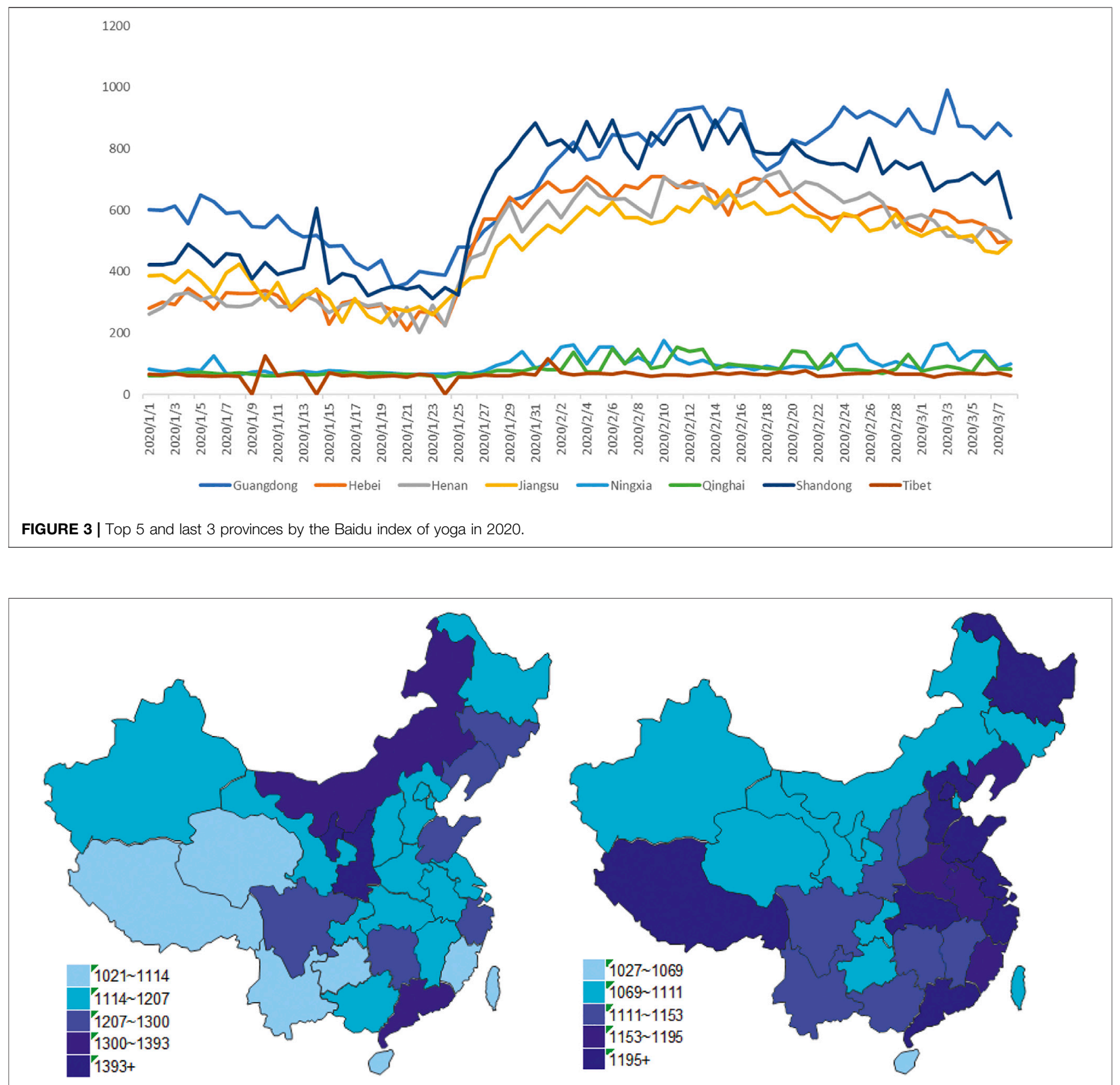

FIGURE 4 | The comparison of the total number of edges between 2011 and 2019.

and, on the other hand, benefited from the improvement of network infrastructure construction.

In addition, with the rapid development of the mobile phone and information technology, the mobile phone starts to become the main channel for information because it is more convenient and easy operating especially when doing exercise following the video on the Internet.

\section{Characteristic Evolution}

Calculating the characteristic of visualized networks, the information is found out including the total number of edges, average degree, average path, diameter, clustering coefficient, and the annual results of density. Given space limitation, it only shows the comparison chart of 2011 and 2019 in Figures 4-9. 


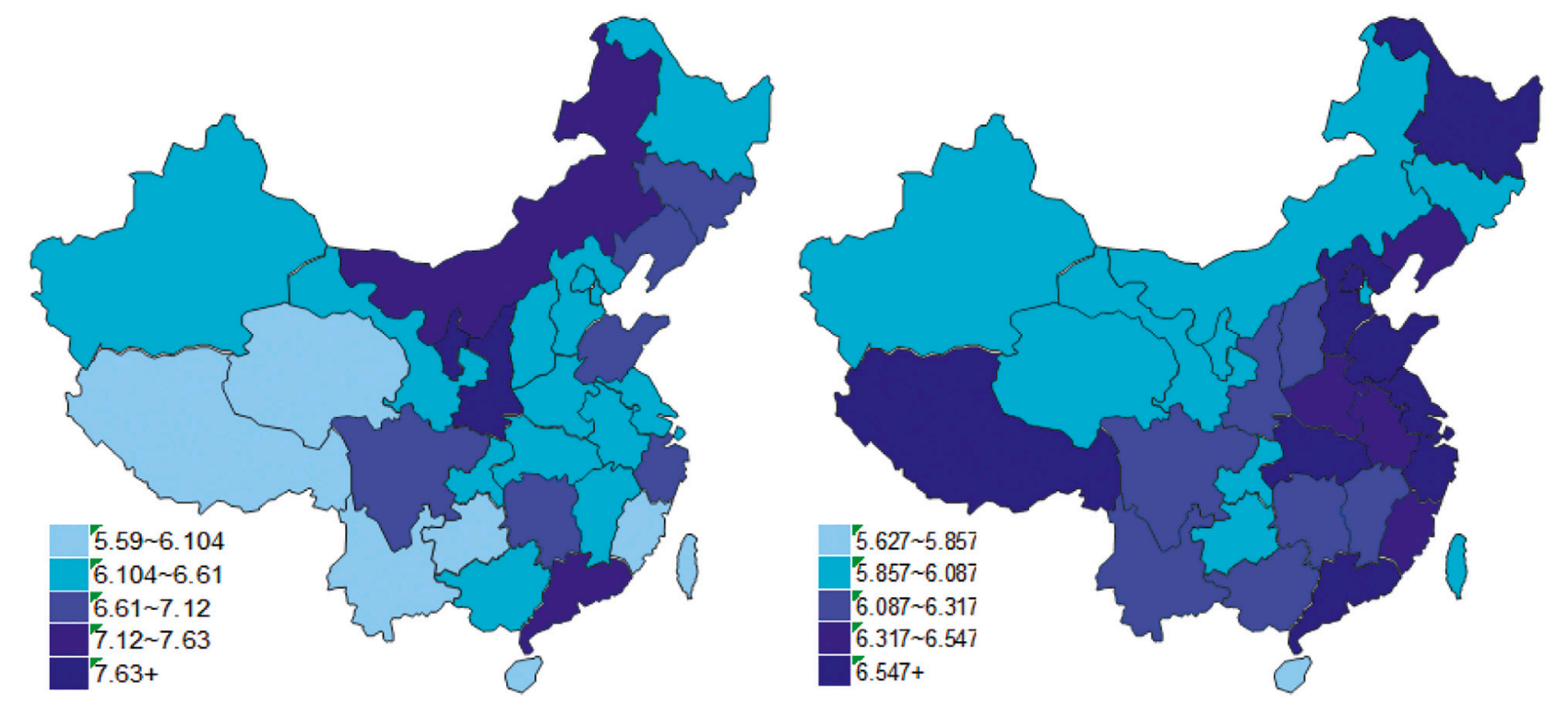

FIGURE 5 | The comparison of average network degree between 2011 and 2019.

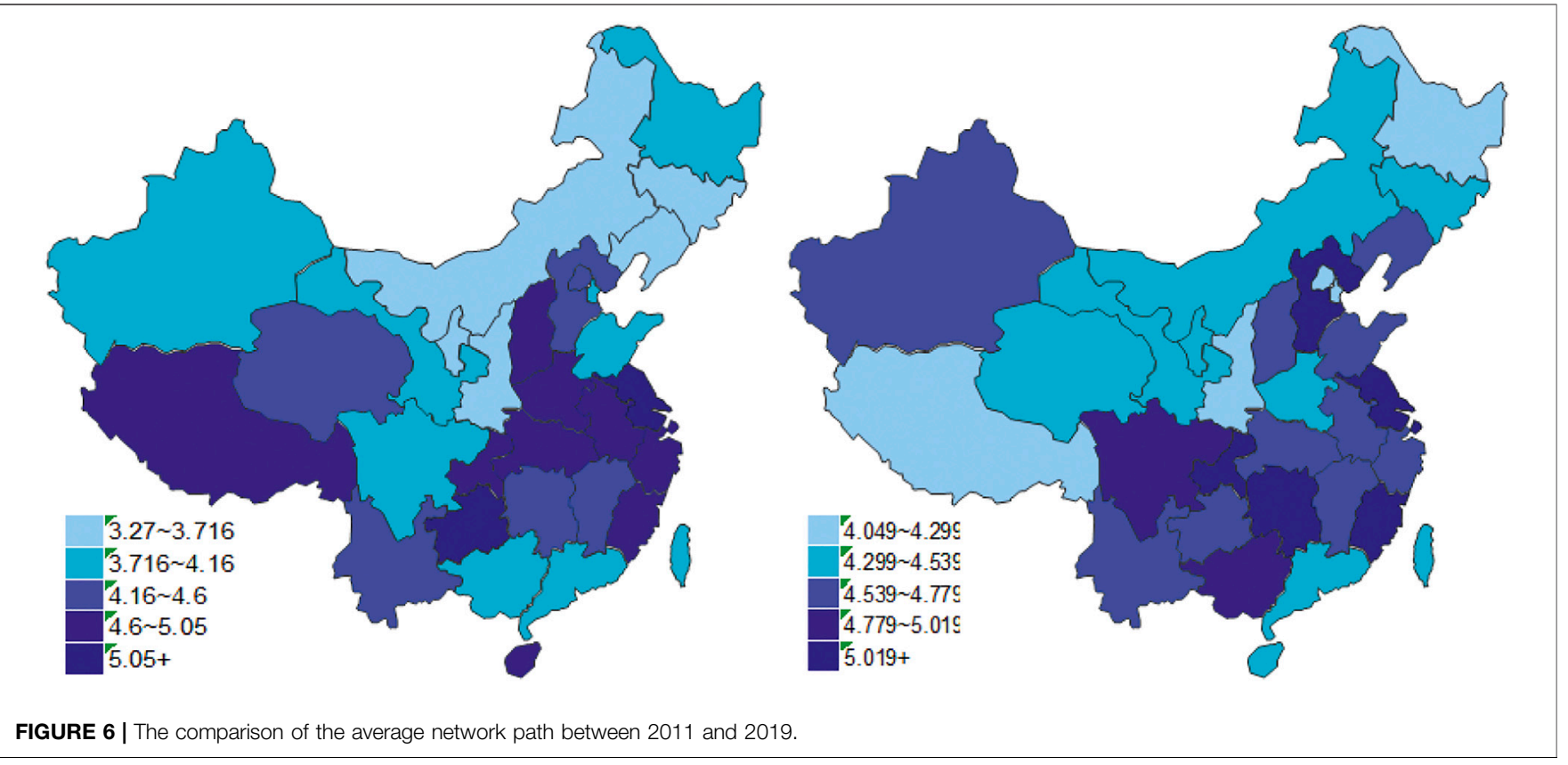

\section{Annual Change Characteristics of Yoga Attention}

1) Geographical distribution characteristics of yoga attention

According to the analysis of relevant charts in 2011, we can find that most of the provinces and cities which pay great attention to yoga fall into the area of ethnic minority settlements or its neighboring provinces and cities, while the central and southwestern regions paid relatively little attention to yoga.
Since 2012, the peaks of attention appeared in Anhui, Hubei, Tibet, Yunnan, Guangxi, Shanxi, Hunan, and other places. Among them, Tibetans pay the greatest attention to yoga in China. In recent years, the attention to yoga in coastal areas has been rising.

We found that the degree of attention to yoga changes over time segments. The local people may search "yoga" a lot at a certain point in time, causing the increase of the index, while after the peak arrives, a sharp decline may occur because the exercise habit of the local people has not been cultivated due to exercises conditions or time limits. At the same time, first-tier cities and 


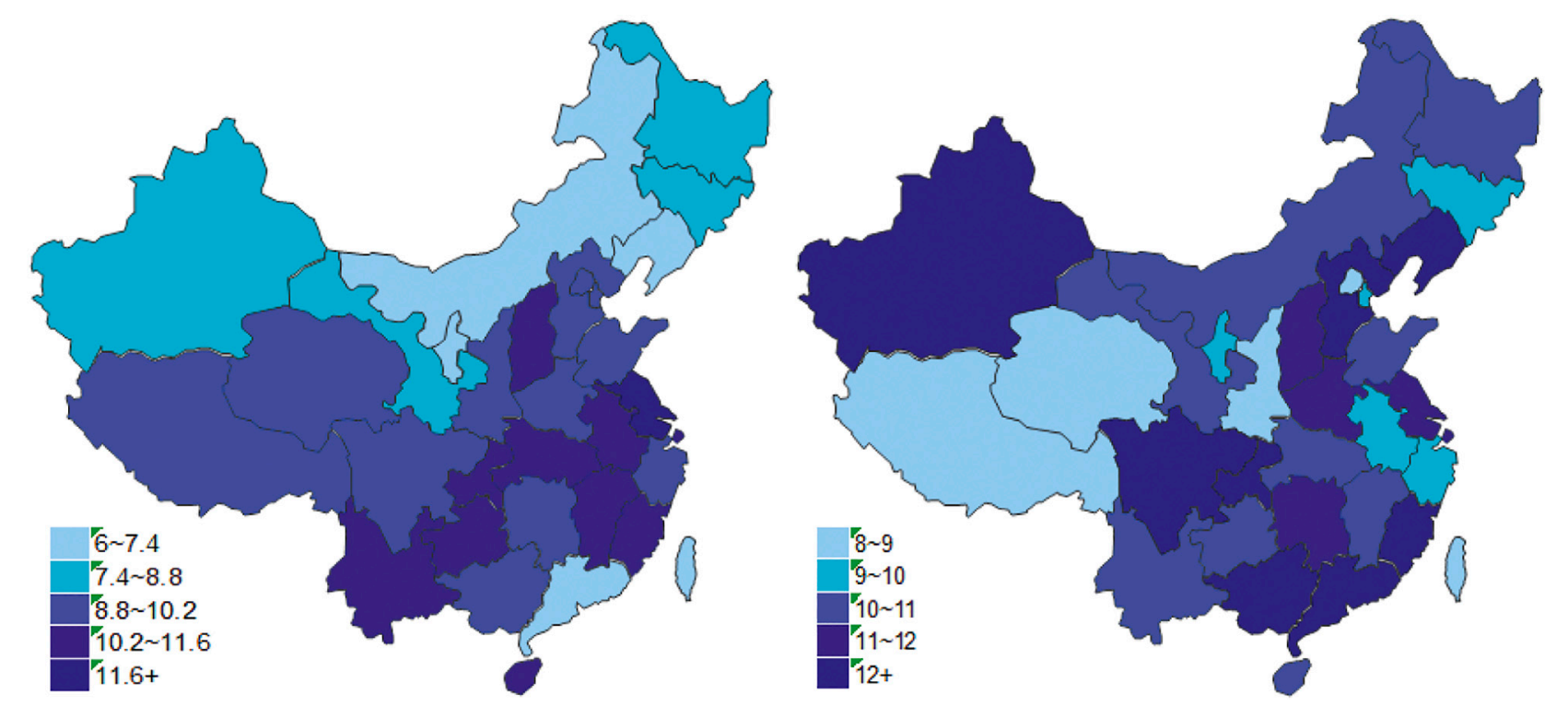

FIGURE 7 | The comparison of average network diameter between 2011 and 2019

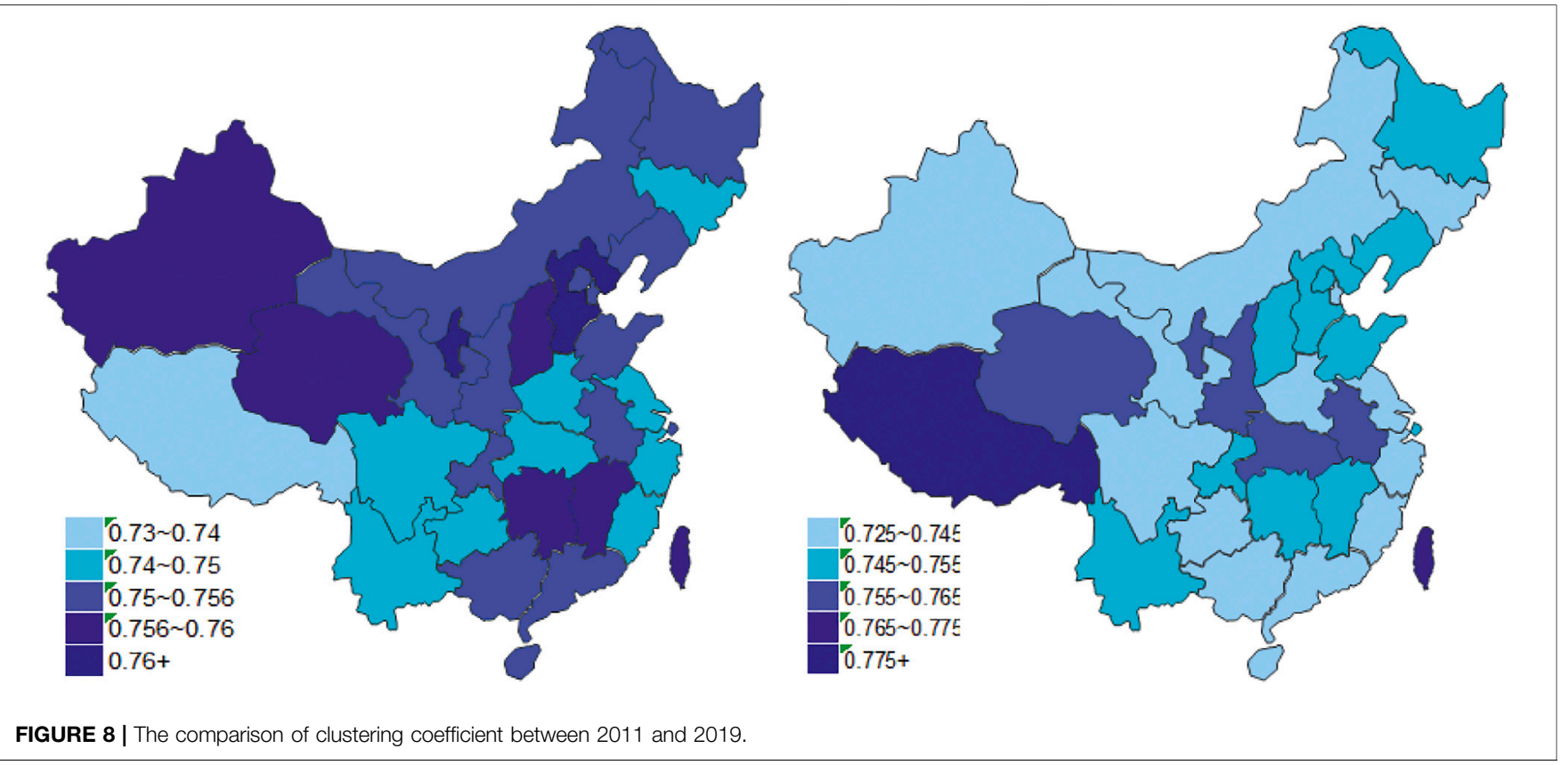

provinces pay less attention to yoga and among them, Shanghai pays comparatively great attention.

\section{2) Temporal characteristics of yoga attention}

Compared with 2011, it is found that the attention to yoga in most provinces has increased in recent years due to the development of yoga industry. In particular, with the development of the Internet, the retention time of information is longer, and the influence of each point of time on the others and the correlation among them are more prominent. Collectively, it is mainly reflected in the following aspects:

(1) The network average degree has improved. That is, as time changes, the average influence of each node increases, and its time correlation is stronger.

(2) The total number of edges is relatively reduced. The reduction in the number of edges indicates that the visible amount of data at the top of the data bar is reduced, which indicates that the peak and bottom of the region's attention that year frequently occurred, meaning greater fluctuations. 

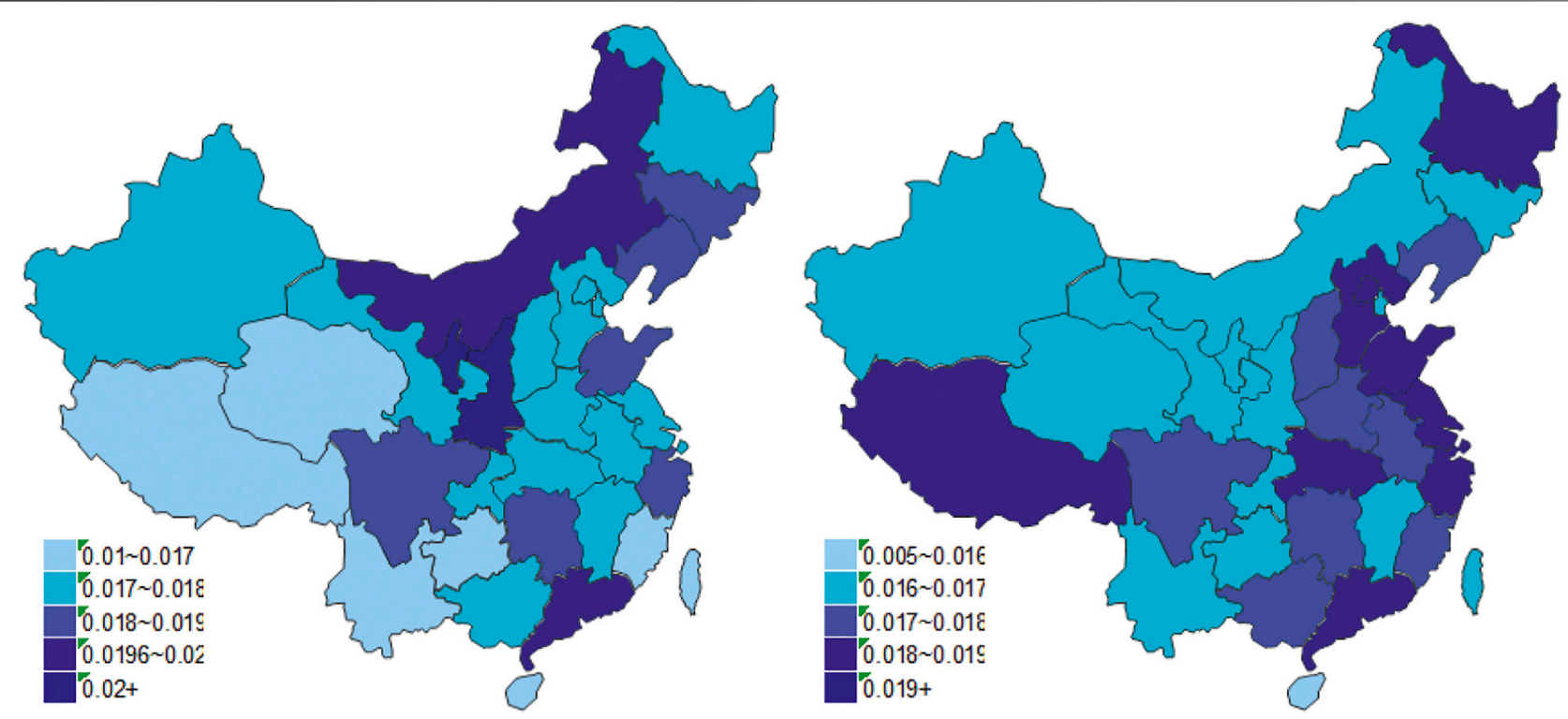

FIGURE 9 | The comparison of network density between 2011 and 2019.
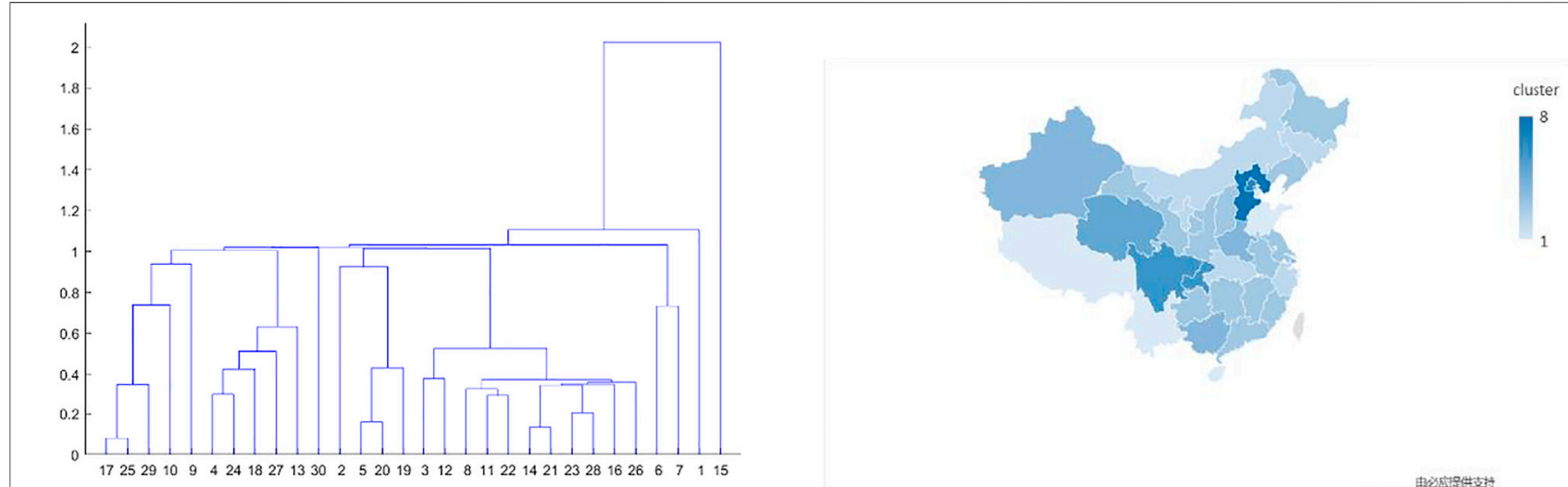

FIGURE 10 | Systematic clustering

(3) The average path and diameter of the network are longer and have been relatively stable in recent years. This shows that network efficiency declines with the passage of time and the rise of emerging technologies.

Relevant information can also be found from the existing literature. With the development of yoga, people start to analyze yoga from various aspects such as theoretical training, method learning, and practical skills. Before 2011, yoga literature focused on theoretical studies, including the fitness effects of yoga and physical education research, the comparison of traditional sports and culture between China and India, the study of religious Buddhism and the interpretation of philosophical thoughts, which did not arouse the public's interest, hence attracted little attention from the public. Nowadays, the promotion of yoga focuses on people's daily life, such as soothing emotions, improving posture, which is the pursuit of people of all ages. News from all aspects attracts more public attention and the development of communication technology speeds up the spread of news in a short period of time, which has led to an increase in the frequency of related searches.

The diversified development of yoga presents different fashion trends at different times. The gimmick marketing could bring huge attention to yoga in a short period of time, but the attention will decrease quickly because the content of related articles is either exaggerated or unprofessional, resulting in a relative decrease in the total number of edges, which directly affects the average path and diameter of the network. 
In this paper, we analyze the cluster data of 31 provinces in China, as shown in Figure 10.

It is found that Hebei, Beijing, and Qinghai are the same cluster; Sichuan and Chongqing are the same cluster; Shanghai, Henan, Xinjiang, and Guangxi are the same cluster; Guangdong, Jiangsu, Fujian, Heilongjiang, Shaanxi, Liaoning, Shanxi, Hunan, Jiangxi, Anhui, Gansu, and Guizhou are the same cluster; Hubei, Zhejiang, Jilin, Neimenggu, and Ningxia are the same cluster; Tianjin, Shandong, Yunnan, Hainan, and Xizang are the same cluster.

\section{CONCLUSION}

Using the complex network to visualize the Baidu search index, we can clearly see the distribution of yoga attention in various provinces and cities and the changes that have occurred in recent years. The development of the network environment has provided a good carrier for the spread of yoga. The development of various public accounts and APPs has made the barriers for yoga practitioners continue to shrink, while the targeted group continues to expand, and the attention of yoga continues to rise. But at the same time, it has also led to the spread of distorted information and the decrease of public's interest in yoga. More importance shall be attached to the implementation of basic and popular tutorials so as to boost the development of yoga in China. The public should also choose wisely and get information from relatively authoritative content. All social organizations such as schools, yoga education institutions, and the local government should formulate the corresponding standards and system for the healthy development of yoga. Firstly, different standards shall be formulated based on the characteristic of different age groups and provide different tutorials and guidance methods for teenagers, middle-aged, and the elderly so as to help them practice yoga reasonably. The second is to promote yoga among the targeted group with practical content and tell them about the physical and mental health effects of yoga. Though the theoretical interpretation of yoga culture is of no interest to the public, its positive effect on various diseases and physical and mental health is obvious for them to understand. The positive function of yoga shall be

\section{REFERENCES}

1. Singleton M. Suggestive Therapeutics: New Thought's Relationship to Modern Yoga. Asian Med Tradition Modernity (2017) 3(1):64-84. doi:10.1163/ $157342107 \times 207218$

2. Wang M. The Study on the Influence of Yoga Body Training on Female College Students' Physical and Mental Health. J Guangzhou Inst Phys Educ (2005) 25(2):84-7. doi:10.4028/www.scientific.net/amr.187.164

3. Satyapriya M, Nagendra HR, Nagarathna R, and Padmalatha V. Effect of Integrated Yoga on Stress and Heart Rate Variability in Pregnant Women. Int J Gynecol Obstet (2009) 104(3):218-22. doi:10.1016/ j.ijgo.2008.11.013

4. Qi J, Zhang Y, and Du Q. Analysis of the Biomechanical Mechanism of Yoga in the Rehabilitation of Patients with Chronic Low Back Pain [J]. Chin J Med Phys (2017) 34(7):748-52. doi:10.3969/j.issn.1005-202X.2017.07.020 publicized to attract more targeted groups. Thirdly, the government should strengthen guidance and supervise relevant online content to avoid distorted and false information. At the same time, the government should encourage the adoption of new social media forms such as short video platforms to facilitate the production of online information in a healthy and upward way. It is also seen from the study of the geographical characteristic of yoga attention that the yoga attention has not been raised to a higher level because all provinces are promoting yoga in their own local areas with little cooperation, causing development barriers due to region limitations, which does not contribute to the development of yoga in China as a whole. All kinds of activities such as offline exercise can be organized to help expand the influence of yoga, giving full play to the advantages of different provinces to raise the attention of yoga.

Visualizing time series data is more intuitive and convenient to analyze the changing trend of data and explore the deep laws and characteristics of the development of the industry. This paper applies the time series visualization method to the study of yoga attention, which helps to grasp the current situation of yoga sports development and propose the policy to guide the future development direction. This study provides a paradigm and sample for the application of time series visualization methods in the field of social science research for further advancement of other analogous studies.

\section{DATA AVAILABILITY STATEMENT}

The datasets presented in this article are not readily available. Requests to access the datasets should be directed to https://index. baidu.com/v2/index.html\#/ and the corresponding authors.

\section{AUTHOR CONTRIBUTIONS}

PL: conceptualization, methodology, software, validation, drawing, and writing; QM: visualization, software; YM: computation, drawing, and writing; JN: visualization, software; JL: investigation; JH: writing-reviewing and editing.

5. He J, Wang G, and Wang H. Meta-analysis of Yoga Intervention for Major Depressive Disorder. J Int Psychiatry (2018) 56(3):432-6. doi:10.1016/ j.jad.2017.02.006

6. Ma K, Liu J, and Fu C. Research Progress on the Interventional Effect and Mechanism of Exercise on Depression. China Sports Sci Technol (2020) 56(No.11):12-24. doi:10.16470/j.csst.2020132

7. Han Y, Liu X, and Xiang H. Clinical Progress of Exercise Therapy for Depression. [J]Sports Res Educ (2020) 35(5):85-90. doi:10.16207/ j.cnki.2095-235x.2020.05.016

8. Pan R, Xu L, and Yao X. Effect of Yoga Breathing on Perioperative Psychology of Lung Cancer Patients. [J]Chinese Gen Pract Nurs (2017) 15(2):2610-1.

9. Li Y, Li Y, and Zeng F. Study on the Application of Yoga Breathing Exercise in Lung Rehabilitation of Patients after Lung Cancer Operation. Nurs Integrated Traditional Chin West Med (2020) 6(10):265-9.

10. Huang M. Research on the Development of Yoga Industry. J Guide Sports Culture (2010) 9:79-81. doi:10.3969/j.issn.1671-1572.2010.09.021 
11. Raghuraj P, and Telles S. Immediate Effect of Specific Nostril Manipulating Yoga Breathing Practices on Autonomic and Respiratory Variables. Appl Psychophysiol Biofeedback (2008) 33(2):65-75. doi:10.1007/s10484-0089055-0

12. Telles S, Naveen KV, and Dash M. Yoga Reduces Symptoms of Distress in Tsunami Survivors in the Andaman Islands [J]. Evid Based Complement Altern Med (2007) 4:503. doi:10.1093/ecam/nem069

13. McCaffrey R, Ruknui P, Hatthakit U, and Kasetsomboon P. The Effects of Yoga on Hypertensive Persons in Thailand. Holist Nurs Pract (2005) 19(4): 173-80. doi:10.1097/00004650-200507000-00009

14. Zhang Y-J, Meng K, Gao T, Song Y-Q, Hu J, and Ti E-P. Analysis of Attention on Venture Capital: A Method of Complex Network on Time Series. Int J Mod Phys B (2020) 34(29):2050273. doi:10.1142/S0217979220502732

15. Cui X, Hu J, Ma Y, Wu P, Zhu P, and Li H-J. Investigation of Stock price Network Based on Time Series Analysis and Complex Network. Int J Mod Phys $B$ (2021) 35:2150171. doi:10.1142/S021797922150171X

16. Zhang Y-J, Meng K, Gao T, Song Y-Q, Hu J, and Ti E-P. Analysis of Attention on Venture Capital: A Method of Complex Network on Time Series. Int J Mod Phys B (2020) 34(29):2050273. doi:10.1142/s0217979220502732

17. Cramer H, Lanche R, and Haller H. A Systematic Review and Meta-Analysis of Yoga for Low Back Pain [J]. Clin J Pain (2013) 29(5):450-60. doi:10.1142/ s0217979220502732

18. Bower JE, Garet D, Sternlieb B, Ganz PA, Irwin MR, Olmstead R, et al. Yoga for Persistent Fatigue in Breast Cancer Survivors. Cancer (2011) 118(15):3766-75. doi:10.1002/cncr.26702

19. Bussing A, Michalsen A, and Khalsa SB. Effects of Yoga on Mental and Physical Health: A Short Summary of Review [J]. Evidence-Based Complement Altern Med (2012) 2012:165410. doi:10.1155/2012/165410

20. Alaguraja K, and Yoga P. Influence of Yogasana Practice on Flexibility Among Obese Adolescent School Boys. Int J Yoga, Physiother Phys Educ (2017) 2:70-1. doi:10.1155/2012/165410

21. Alaguraja K, and Yoga P. Impact of Yogic Package on Body Mass index Among Obese People. Int J Phys Educ Exerc Sports (2019) 1:4-6.

22. Alaguraja K, and Yoga P. Combination of Naturopathy and Yoga on VO2 Max Among Hypertensive Patient. Indian J Public Health Res Dev (2020) 11:4.

23. Danhauer SC, Addington EL, Sohl SJ, Chaoul A, and Cohen L. Review of Yoga Therapy during Cancer Treatment. Support Care Cancer (2017) 25:1357-72. doi:10.1007/s00520-016-3556-9

24. Cramer H, Lauche R, Klose P, Lange S, Langhorst J, and Dobos GJ. Yoga for Improving Health-Related Quality of Life, Mental Health and Cancer-Related Symptoms in Women Diagnosed with Breast Cancer. Cochrane Database Syst Rev (2017) 1:CD010802. doi:10.1002/14651858.CD010802.pub2

25. Park CL, Finkelstein-Fox L, Groessl EJ, Elwy AR, and Lee SY, Exploring How Different Types of Yoga Change Psychological Resources and Emotional WellBeing across a Single Session. Complement therapies Med (2020) 49:102354. doi:10.1016/j.ctim.2020.102354

26. Novaes MM, Palhano-Fontes F, Onias H, Andrade KC, Lobão-Soares B, Arruda-Sanchez T, et al. Effects of Yoga Respiratory Practice (Bhastrika Pranayama) on Anxiety, Affect, and Brain Functional Connectivity and
Activity: A Randomized Controlled Trial. Front Psychiatry (2020) 11:467. doi:10.3389/fpsyt.2020.00467

27. Sharma N. The Yoga for Physical and Mental Health - Can Possibly Aid in Prevention and Management of COVID19 Infection? Dev Sanskriti Interdis Internat $J$ (2020) 16:22-31. doi:10.36018/dsiij.v16i.161

28. Zhu P, Wang X, Li S, Guo Y, and Wang Z. Investigation of Epidemic Spreading Process on Multiplex Networks by Incorporating Fatal Properties. Appl Math Comput (2019) 359:512-24. doi:10.1016/j.amc.2019.02.049

29. Firestone K, Carson J, Mist S, Carson K, and Jones K. Interest in Yoga Among Fibromyalgia Patients: An International Internet Survey. Int J yoga Ther (2014) 24:117-24. doi:10.17761/ijyt.24.1.p810750h1r1832q2

30. Johnson CC, Taylor AG, Anderson JG, Jones RA, and Whaley DE. Feasibility and Acceptability of an Internet-Based, African Dance-Modified Yoga Program for African-American Women with or at Risk for Metabolic Syndrome. J Yoga Phys Ther (2014) 4:1000174. doi:10.4172/21577595.1000174

31. Hu J, Xia C, Li H, Zhu P, and Xiong W. Properties and Structural Analyses of USA's Regional Electricity Market: A Visibility Graph Network Approach. Appl Math Comput (2020) 385:125434. doi:10.1016/j.amc.2020.125434

32. Chen X, Gong K, Wang R, Cai S, and Wang W. Effects of Heterogeneous Selfprotection Awareness on Resource-Epidemic Coevolution Dynamics. Appl Math Comput (2020) 385:125428. doi:10.1016/j.amc.2020.125428

33. Pan L, Yang D, Wang W, Cai S, Zhou T, and Lai YC. Phase Diagrams of Interacting Spreading Dynamics in Complex Networks [J]. Phys Rev Res (2020) 2(2):023233. doi:10.1103/physrevresearch.2.023233

34. Pan L, Wang W, Cai S, and Zhou T. Optimal Interlayer Structure for Promoting Spreading of the Susceptible-Infected-Susceptible Model in Two-Layer Networks [J]. Phys Rev E (2019) 100:022316. doi:10.1103/ physreve.100.022316

Conflict of Interest: QM was employed by Beijing Jingkong Technology Co., Ltd.

The remaining authors declare that the research was conducted in the absence of any commercial or financial relationships that could be construed as a potential conflict of interest.

Publisher's Note: All claims expressed in this article are solely those of the authors and do not necessarily represent those of their affiliated organizations, or those of the publisher, the editors, and the reviewers. Any product that may be evaluated in this article, or claim that may be made by its manufacturer, is not guaranteed or endorsed by the publisher.

Copyright $\odot 2021$ Li, Miao, Meng, Ning, Long and Huang. This is an open-access article distributed under the terms of the Creative Commons Attribution License (CC $B Y)$. The use, distribution or reproduction in other forums is permitted, provided the original author(s) and the copyright owner(s) are credited and that the original publication in this journal is cited, in accordance with accepted academic practice. No use, distribution or reproduction is permitted which does not comply with these terms. 